\title{
Highly Dexterous 2-module Soft Robot for Intra-organ Navigation in Minimally Invasive Surgery
}

\author{
Haider Abidi, Giada Gerboni, Margherita Brancadoro, Alessandro Diodato, Matteo Cianchetti, Helge Wurdemann, \\ Kaspar Althoefer and Arianna Menciassi
}

\begin{abstract}
This paper describes the design, fabrication and modeling of a highly dexterous 2-module soft robot for minimally invasive surgery (MIS). Some surgical interventions, like the Total Mesorectal Excision (TME), traditional laparoscopes lack the flexibility to safely maneuver and reach difficult surgical targets. This work proposes a soft robotic approach using flexible fluidic actuators (FFAs), allowing highly dexterous and inherently safe navigation. Dexterity is provided by an optimized design of fluid chambers within the robot modules. Safe physical interaction is ensured by fabricating the entire structure by soft and compliant elastomers, resulting in a squeezable 2-module robot. An inner free lumen/chamber along the central axis serves as a guide of flexible endoscopic tools. A constant curvature based inverse kinematics model is also proposed, providing insight into the robot capabilities. Additionally, experimental tests in a surgical scenario are reported using a cadaver model, demonstrating the robot capabilities in comparison to standard systems used in a realistic MIS environment.
\end{abstract}

\section{INTRODUCTION}

M INIMALLY invasive surgery (MIS) is quickly becoming the norm in surgical interventions, with an increasing portion of surgical procedures being performed via laparoscopy. Open surgery is progressively being reduced to emergency conditions, where operation planning is not possible [1]. From the patient point of view, the advantages of MIS may outweigh the complications by a significant margin. However, for surgeons the difficulties encountered in properly maneuvering instruments in a constrained and sensitive workspace such as the human body's internal organs poses significant limitations [2]. A typical laparoscopic tool workspace is in the form of a cone (or multiple cones) having the vertex at the entry point, i.e. the incision (Fig. 1). The entry point, provided by the trocar, serves as a fulcrum for maneuvering rigid instruments. The surgeon can maneuver a standard rigid laparoscopic instrument with 4 degrees of freedom (DOFs) by longitudinally sliding the rigid tool into the trocar and pivoting around the fulcrum [3]. This level of

Haider Abidi, Margherita Brancadoro, Alessandro Diodato, Matteo Cianchetti and Arianna Menciassi are with the Biorobotics Institute, Scuola Superiore Sant'Anna, Viale Piaggio, 34, Pontedera (PI), 56025 Italy. \{s.abidi, m.brancadoro, a.diodato, m.cianchetti, a.menciassi\}@santannapisa.it.

Giada Gerboni is with Stanford University, Center for Design Research (CDR), 424 Panama Mall, Stanford, CA 94305-3030 USA. ggerboni@stanford.edu

Helge Wurdemann is with University College London, Department of Mechanical Engineering, London WC1E 7JE. h.wurdemann@ucl.ac.uk

Kaspar Althoefer is with Queen Mary University of London, School of Engineering and Materials Science, London E1 4NS, k.althoefer@qmul.ac.uk

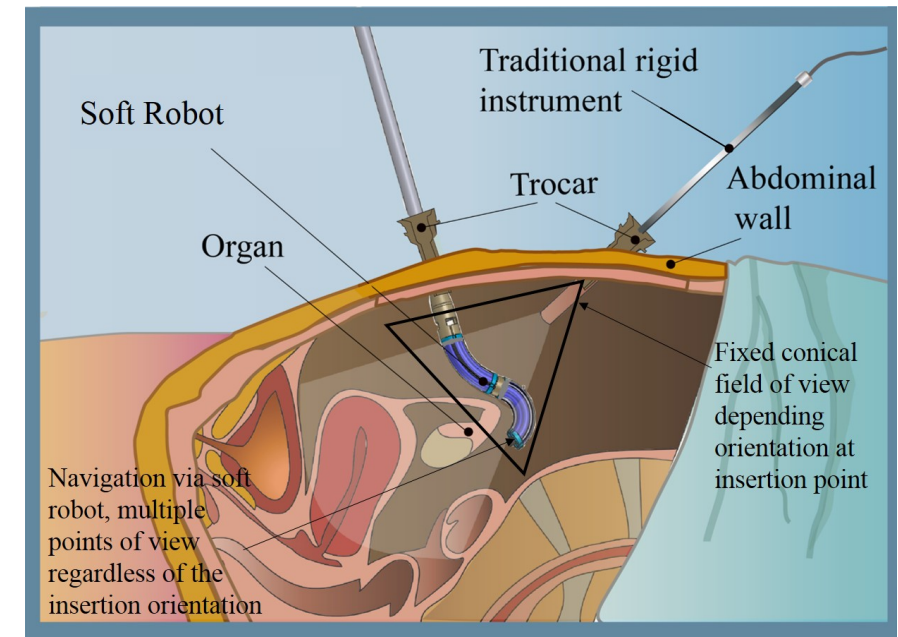

Fig. 1. Illustration of the potential advantages of a soft robotic device in terms of workspace in comparison to a rigid laparoscopic instrument

maneuverability limits the access to all areas of the surgical site. This is especially true in the case when multiple organs are present between the entry point and the target site. Hence the actual workspace of a rigid instrument is greatly reduced. In the presence of obstacles, the pivoting of the shaft at the fulcrum may be impeded such that even if the target is reached, no further maneuvers are possible. This dependence on the position and orientation of the instrument at the insertion point clearly results in very poor manipulability.

Highly dexterous and flexible tools are therefore greatly welcomed by surgeons performing MIS procedures. In the case of an endoscope, high dexterity may translate into multiple points of view of the surgical site, thus providing the surgeon with more information about the target site. Examples of demanding procedures in terms of advanced instrument flexibility are the posterior and lateral total mesorectal excision (TME) operations. Clinicians performing TMEs have reported difficulties related to poor maneuverability of the current rigid instruments [4] [5]. Laparoscopic instruments with additional DOFs at their tip may be provided to overcome such limitation. Some examples of such enhanced laparoscopic instruments already exist. The robotically actuated Endowrist of the DaVinci System, which combines a special wrist at the tip of a shaft moved by the teleoperated robotic system [6], is one of them. Numerous other flexible MIS platforms, 
though still at research level, have been developed such as the i-Snake [7] and [8]. The SurgiBot by Transenterix is another valid example of flexible instrumentation for MIS which is already available in the market. It uses a hybrid of manual user actuation along with robotic assistance [9] to perform its tasks. Surgical manipulators from hyper-elastic materials also exist such as [10], or as multi-backbone robots [11] or cannula robot [12], [13], [14].

Given the enhanced tip dexterity of these instruments, the requirement of safely navigating tools around organs to reach remote areas and maintaining residual maneuvering abilities to accomplish different tasks is still a challenge. Alongside the need for high dexterity, the surgical environment is particularly demanding in terms of safety with respect to contact with the surrounding tissues an organs. Hence, high dexterity and safe interaction with the encountered organs are both essential for an efficient MIS tool.

The combined effect of flexibility and safety is embodied by soft robotics. This branch of robotics uses flexible actuators constructed with soft and highly compliant materials to create intrinsically safe tools [15], [16], [17], [18] [19] when used in traditional applications [20]. The so called flexible fluidic actuators (FFAs) using silicone based structures are soft robotic actuators which are especially suitable for medical purposes. A configuration with three pairs of inflatable chambers, radially arranged around a central axis, produces an elastomeric module capable of bending in every direction and also elongating longitudinally depending on the pressure in the chambers [21]. The chambers act as actuators that generate up to four motion primitives (MPs). The term MPs are used here instead of the traditional DOF since it is difficult to define the degrees of freedom in soft robots, which theoretically have infinite DOF since it is composed of a continuum-like structure. Three MPs describe the pure bending motion in the three dimensional space of the actuator due to the inflation of one chamber at a time (Fig. 2-a). The fourth MP is the longitudinal translation of the actuator tip, representing elongation of the complete structure because of the simultaneous inflation of all chambers (Fig 2-b). If more modules are connected in series, a highly articulated structure is generated [22]. These soft actuators show great potential for surgical applications. They can employ inert fluids, potentially biocompatible materials and nonabrasive surfaces, which are ideal for use within the human body [23]. Additionally, due to their high compliance, they can produce large displacements with low actuation pressures. On the other hand, when deflated, they can easily be squeezed and made to pass through narrow cavities [24]. Because of these favorable characteristics, soft robots employing FFAs have already been applied to the medical field [15]. The STIFF-FLOP robot described in [25] is an example of pneumatic actuated manipulator which combines the flexibility of FFAs with means of controlling stiffness by an integrated granular jamming-based mechanism. However such a version of the STIFF-FLOP robot had a diameter of $35 \mathrm{~mm}$ making it incompatible with traditional trocars, and did not have a free lumen.

This work is focused on developing a highly flexible soft robot, intended to serve as an access channel for the typical
MIS instruments, and help approach remote or difficult-toreach areas of the body during laparoscopic procedures. The robot allows safe navigation around organs and, thanks to an internal free lumen, it can be used to feed instruments such as surgical tools for endoscopes. It can also house endoscopic sensors, such as a camera which can be fixed on its tip, as in the case of this work. The aim of this work is developing an intrinsically safe robot which provides unprecedented dexterity in the constrained MIS environment, while keeping the dimensions of typical MIS instruments.

The requirements for the development of the proposed system are detailed in Section II, while the system design is described in Section III. Section IV explains the manufacturing process of the modules composing the soft robot. Section V is dedicated to the modeling of the soft robot, in order to estimate its capabilities. Section VI is dedicated to test the performance and also sheds light on a surgical use case scenario, followed by the discussion.

\section{REQUIREMENTS}

In lieu of the above mentioned considerations, the soft robot has been designed with the objective of producing a highly flexible guide for surgical instruments/devices, which ensures safe interaction with organs during maneuvering. The design was carried out considering the following requirements:

$\mathrm{R}$ 1: The device has to be compatible with traditional trocars, thus a diameter smaller than $15 \mathrm{~mm}$ is required. This also means that the employed FFAs must not deform radially (e.g. avoid chamber lateral expansions, ballooning, etc.) during operation. This would reduce risk of tearing and also ensure efficient use of fluidic actuation.

R 2: The robot should be able to adjust the position and direction of the end-effector while keeping the shaft stationary; thus more than 4 MPs (3 for plane bending and 1 for elongation) are necessary to enable the required dexterity. This translates into more than one module, each integrating 4MPs.

$\mathrm{R}$ 3: The soft modules must be compliant in order to allow squeeze-ability of the deflated robot from tight spaces (especially in case of an emergency).

$\mathrm{R}$ 4: A free lumen of about $4-5 \mathrm{~mm}$ is required for feeding surgical tools (e.g. laparoscopic graspers, RF tools, etc.), or to house the wires of a laparoscopic camera which can be fixed at the tip.

\section{SOFT Robot DESIGN}

The illustration in Fig. 1 shows the complete soft robot, which is composed of two identical pneumatically actuated modules (Fig. 2). The robot is attached to a rigid shaft, which is hollow and serves as a support during the MIS procedure. This rigid shaft also connects the flexible robot to a pressure control system. The shaft can be positioned and maneuvered at the insertion point by a medical personnel or by a robot.

The two modules are identical. Both are capable of omnidirectional bending and elongation motion ( $\mathrm{R} 2$ ) in response to pressure applied to their inner chambers (Fig 2-a and b). The modules are connected to each other through thin $3 \mathrm{D}$ printed 
rigid connectors attached to the ends of the modules. The first module, called the base module, is also rigidly connected to the shaft. The shaft contains the six pneumatic pipes connecting each module's chamber to the external pressure system. The pneumatic pipes of the second module, called the tip module, are guided through the lumen of the base module. Hence while lumen of the tip module is completely free, the base module lumen is partially taken. However there is still enough space to pass additional equipment through, such as a camera module of $3 \mathrm{~mm}$ diameter.

Fig. 2-c shows a detailed view of a single module, which is $50 \mathrm{~mm}$ in length and $14.5 \mathrm{~mm}$ in external diameter, making it suitable for MIS application by employing standard trocars ( $\mathrm{R}$ 1). The module contains three pairs of cylindrical elongating only chambers [26], each measuring $3 \mathrm{~mm}$ in diameter. A cylindrical shape ensures efficient use of fluid pressure for producing the required elongation. These chambers are lined with a minimum pitch thin in-extensible thread in a tight helical wound (Fig. 2-c, inset). The helix pitch of the thread plays a fundamental role. It determines the space left for the silicone to expand radially under pressure. The minimum helix pitch design means that the incorporated thread turns are in contact with each other, resulting in maximizing chamber elongation [27].In this way pressurization effects are not lost through internal expansions or through friction between the chambers and the structure (as in earlier designs [28]). Therefore the actuation has been optimized to provide only the required longitudinal elongation of the chambers.

As visible in Fig. 2, six chambers make up the module. There are a couple of chambers for each bending MP. The choice of using a double cylinder configuration (i.e. a pair of simultaneously activated chambers) has a twofold advantage. On one hand, it helps to meet the free central lumen requirement ( $\mathrm{R} 4)$. The bending force is proportional to the product of the pressure in the chambers and the cross sectional area of the chamber. Given the diameter of the module and the free lumen, there is no space available for chambers of larger dimensions, as can be seen in Fig. 2. Using a double cylinder configuration increases the bending force by almost a factor of two. Hence the couple of chambers have an equivalent effective area of a single, yet larger in diameter, chamber. That would have taken up more space towards the central axis. Secondly, the double cylinder configuration provides a more stable bending motion of the entire module owing to a distributed effect of pressure on the entire module.

These design features (i.e. reducing radial expansion of the modules, use of a two-chamber expansion unit, and embedding all chambers in a single silicone body (R 3)) result in a smooth constant curvature bending of the module when pressurized (Fig 2-a). This feature has been observed experimentally and is used when modeling the kinematics, as discussed in the next section. The constant curvature behavior of the STIFF FLOP module can be measured by dragging a small magnetically tracked sensor along the lumen of the module and observing its trajectory in the 3D space (Fig. 3). An internal pipe connects the two chambers related to the same bending motion; making a pair of chambers essentially one chamber. Each pair is hence supplied by just one pipe from the central system pressure


b. Longitudinal elongation

\section{c. Module design}

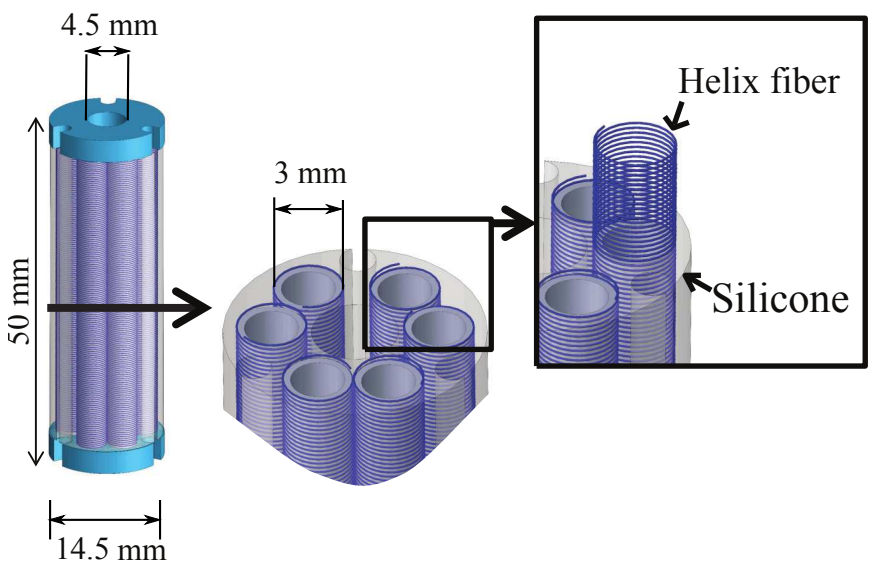

Fig. 2. Single module behavior and design. a) Constant curvature deformation due to the increased air pressure in one pair of chambers (highlighted in dark blue). b) Longitudinal elongation due to the application of equal pressures to all the chambers. c) Illustration of the module design: main dimensions, view of the structure of the internal chambers and close up of the exploded view of a chamber.

control. Two end caps made of a stiffer silicone (depicted in blue in Fig. 2-c) are added to the top and bottom part of the module, hermetically sealing the chambers. The bottom of the module is designed to embed the three pipes for the pneumatic pressure supply, as described in the next section.

\section{SOFT ROBOT FABRICATION}

The manufacturing of the module consists of several silicone molding steps. Firstly, the mold for the chambers is prepared by winding an in-extensible polyester thread around a 3D printed cylinder. This cylinder is composed of three assembled parts, an inner core and two side parts (Fig. 4-a). Six molds are positioned into a module mold (Fig. 4-b, step I). Uncured silicone (EcoFlex 0050, Smooth On) is poured into the mold and left to cure at room temperature. After the silicone has been completely cured the chamber molds are removed, starting from the core and then sliding out the other two side parts, blue and green. This disassembly of the chamber molds allows the loosening of the chamber molds within the cured body, easing the removal and keeping the thread in place. At this point the thread is only partially embedded in the bulk of silicone body of the module, as shown in the picture of a cut module in Fig. 4-b. Another layer of silicone is added to the inner side of the chambers, completely integrating the thread within the body. To do so, thinner rods are inserted 

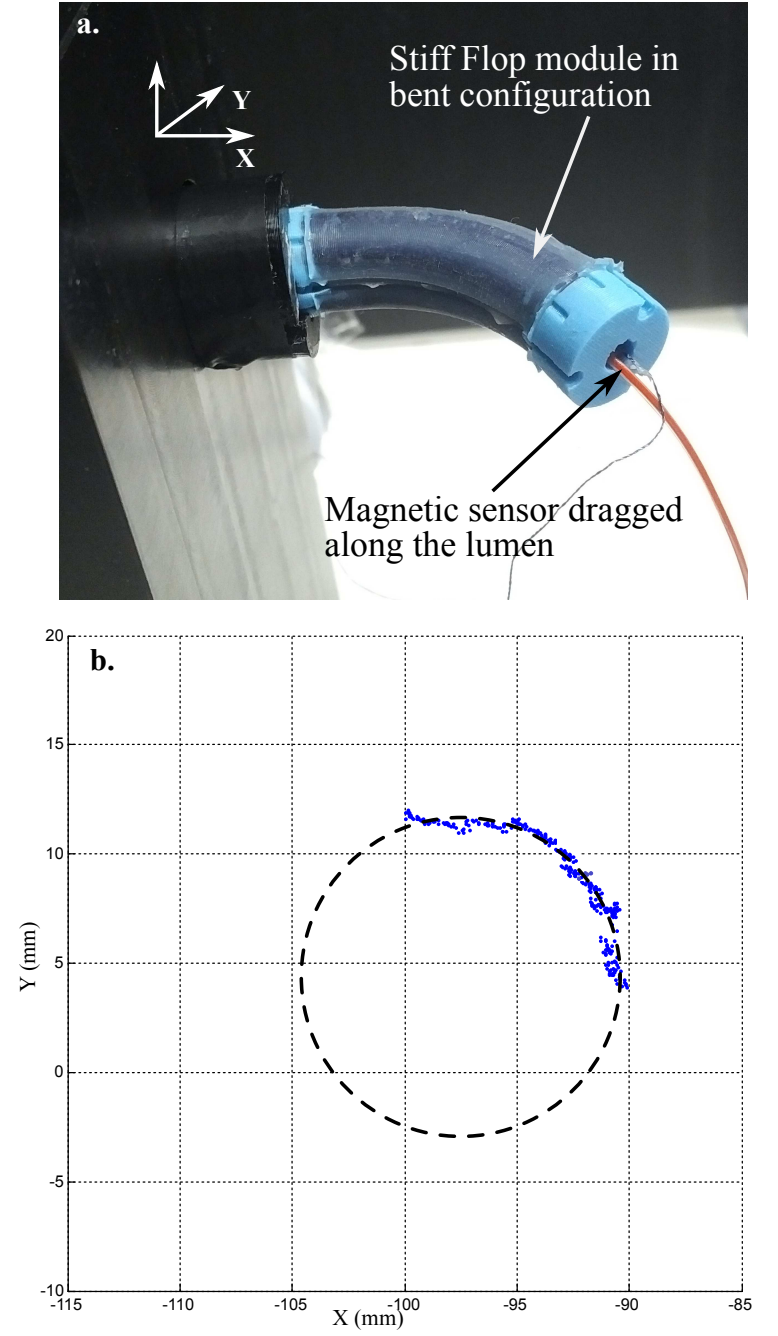

Fig. 3. a) Magnetic sensor shown in the lumen. The sensor was dragged along the lumen to record the shape of the module in a bent configuration. b) Tracked points (in blue) collected by the magnetic sensor. A dotted circle is superimposed over the points, showing a constant curvature.

into the chambers and the partially complete body is placed in a new mold. A second curing step (Fig. 4-b, step II)) is therefore performed by pouring the same uncured silicone into the remaining free space between the partially complete module and the new mold. This step completes the body of the module.

The next phases (Fig. 4.b, step III and IV) deal with the sealing of the chambers and connecting the cylinders of the same chamber pair to one pressure input. For these two phases, a hard silicone (Smooth Sil 950,Smooth On) is used as end stubs. During step III, three actuation pipes, one for each couple of chamber, are fixed at the bottom of the module. They serve as the pressure interface to the external actuation source. The internal pipes connecting the double chambers are added to the top of the module (Fig. 4-b, step IV) and sealed with the same hard silicone of step III. This manufacturing chain results in a complete module (Fig. 4.c). The final weight of each module comes to about $8.5 \mathrm{~g}$.

\section{Configuration/Kinematic Model}

Definition of the kinematics allows a direct mapping between the actuator states, or the lengths of the actuators, and the coordinate space position of the end effector, removing the influences of the sensors themselves. It also enables simulating the capabilities of the robot and ultimately effectively controlling it, without the use of position sensors [29], [24] which may influence the soft robot performances [30], [31], [32]. Based on the design of the module and for the sake of simplification, the kinematics are based on the constant curvature (CC) assumption [33]. The kinematics mapping can be broken into two parts, one from the states of the robot (length of chambers in this case) to arc parameters and the other from the arc parameters to the position of the robot in the coordinate system. The mappings are defined for each module, and hence can be used for a multi-module robot. It is also assumed that the elongations of the chambers are proportional to the supplied pressure [21].

\section{A. Kinematics}

Under the CC assumption, the orientation of the robot can be defined by the use of three parameters $\kappa, \phi$ and $l$ which represent curvature, out of plane motion of the robot and the length of the robot back bone respectively. $\kappa$ is the inverse of the radius of curvature $r$. These parameters are called as the configuration parameters (Fig. 5). Hence the end effector position with respect to the base of the robot can be represented by a transformation given as

$$
T=\left[\begin{array}{cc}
R & u \\
0 & 1
\end{array}\right]
$$

where

$$
\begin{gathered}
R=\left[\begin{array}{ccc}
C^{2} \phi(C \kappa s-1)+1 & S \phi C \phi(C \kappa s-1) & C \phi S \kappa s \\
S \phi C \phi(C \kappa s-1) & C^{2} \phi(1-C \kappa s)+C \kappa s & S \phi S \kappa s \\
C \phi S \kappa s & -S \phi S \kappa s & C \kappa s
\end{array}\right] \\
u=\left[\begin{array}{c}
\frac{C \phi(1-C \kappa s)}{S \phi(1-C \kappa s)} \\
\frac{S_{\kappa s}^{\kappa}}{\kappa}
\end{array}\right]
\end{gathered}
$$

$C$ and $S$ represent $\cos$ and $\sin$ respectively. In order to obtain the shape of the complete backbone, the length $l$ has been parameterized by a variable $s=[0, L]$ in equation (1). However, equation (1) is not enough to complete the forward mapping. A relation between the configuration parameters and the actuator states also needs to be established for this purpose. As per [33], for a module with 3 actuators placed at $120^{\circ}$ to each other and a distance $d$ from the central axis, the relation between the lengths of individual chambers $\left(l_{1}, l_{2}, l_{3}\right)$ and the configuration parameters $(\kappa, \phi, l)$ can be given by the equations (2),(3) and (4). These equations, along with equation (1), complete the forward kinematics model.

$$
\begin{gathered}
l=\frac{l_{1}+l_{2}+l_{3}}{3} \\
\phi=\tan ^{-1}\left(\frac{\sqrt{3}\left(l_{2}+l_{3}-2 l_{1}\right)}{3\left(l_{2}-l_{3}\right)}\right)
\end{gathered}
$$


a. Chamber mold assembly

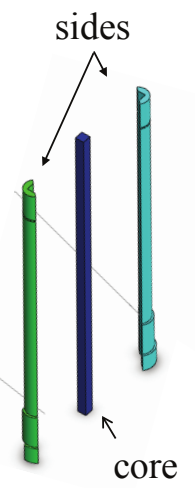

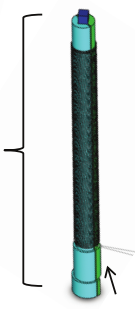

helix thread b. Silicone casting
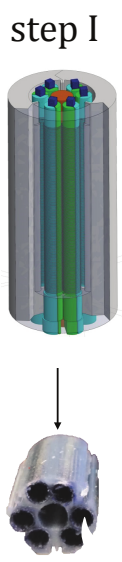



c. Finalized module


Fig. 4. Module fabrication steps. a) The mold for the chambers is prepared by assembling a support structure for the chambers and then winding a helical thread around the mold. b) Four steps of the silicone casting process: first the body of the module is prepared (Step I and II) followed by the module sealing at the top and bottom end (step III and IV). c) The finalized module.

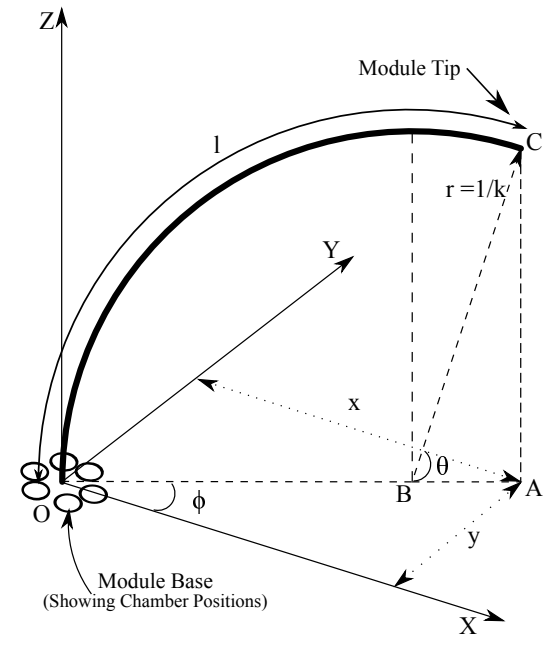

Fig. 5. Configuration parameters and their relation to the coordinate system

$$
\kappa=\frac{2 \sqrt{l_{1}^{2}+l_{2}^{2}+l_{3}^{2}-l_{1} l_{2}-l_{1} l_{3}-l_{2} l_{3}}}{d\left(l_{1}+l_{2}+l_{3}\right)}
$$

\section{B. Inverse Kinematics}

The inverse kinematics is based on the analytical calculation of the configuration parameters $\kappa, \phi$ and $l$ from the end effector position in the coordinate plane. The calculation of lengths from the configuration parameters then completes the inverse mapping.This second step is relatively straightforward. If the plane of bending is known, the lengths of the individual chambers can be directly found because they form parts of a concentric circle about the center of curvature. However, identifying the configuration parameters is challenging, since there may be many configurations ending at the same point. Nevertheless, this issue can also be resolved by defining the plane of bending first. As a single module can produce only planar bending, once the plane of bending is defined, there is only a single curvature and length that will define the end point. This approach is similar to the one used in [34]. For multiple modules, the planes of each module is identified by using the tip position with respect to the respective base position.

The plane of bending is identified by the angle $\phi$. This angle can be found by using the projection of the end effector position on the X-Y plane (Fig. 5). Hence it can be expressed as equation (5)

$$
\phi=\tan ^{-1}\left(\frac{y}{x}\right)
$$

Consequently, considering Fig. $5 \phi$ can be expressed as

$$
\cos \phi=\frac{O X}{O A}=\frac{x}{\sqrt{x^{2}+y^{2}}}
$$

The next step is the identification of the curvature and the length of the manipulator. From Equation 1, the expression of tip coordinate positions are known in the form of $u$. Replacing the value of $O X$ as value of $x$ and rearranging we obtain

$$
\sqrt{x^{2}+y^{2}}=\frac{1-\cos \kappa s}{\kappa}
$$

Add the expression of $z$ (recall from $u$ in equation 1) to the above and rearrange to obtain the expression for $\kappa$ as

$$
\kappa=\frac{2 \sqrt{x^{2}+y^{2}}}{x^{2}+y^{2}+z^{2}}
$$

The length can be found by using the angle subtended by the $\operatorname{arc} \theta$. Recall that, for the CC assumption, $l=r \cdot \theta$. If $B$ is the center of curvature, from Fig. 5 the angle $\theta$ can be expressed as

$$
\cos (\pi-\theta)=\frac{B A}{B C}=\frac{\sqrt{x^{2}+y^{2}}-r}{r}
$$






Fig. 6. Illustration of a few key tip positions of the soft robot using the constant curvature model, keeping the base module position fixed.

It must be noted that in case the $z$ is negative, the value of $\theta$ has to be deducted from $2 \pi$. Rearranging and substituting $r$ by $\kappa$, the expression for $\theta$ can be expressed as

$$
\begin{array}{r}
\theta=\cos ^{-1}\left(1-\kappa \sqrt{x^{2}+y^{2}}\right) \forall z>0 \\
\theta=2 \pi-\cos ^{-1}\left(1-\kappa \sqrt{x^{2}+y^{2}}\right) \forall z \leq 0
\end{array}
$$

Equations 5, 7 and 9 provide the configuration parameters from the coordinate axis positions. Once the configuration parameters are set, the lengths of each chamber can be found. As mentioned before, these chamber lengths will form concentric circles Hence the lengths can be related to the length of the backbone $l$ by the following equations:

$$
\begin{aligned}
& l_{1}=l-\theta d \cos \phi_{1} \\
& l_{2}=l-\theta d \cos \phi_{2} \\
& l_{3}=l-\theta d \cos \phi_{3}
\end{aligned}
$$

where $\phi_{1}=90-\phi, \phi_{2}=210-\phi$ and $\phi_{3}=330-\phi$. Thus, if the tip position is available for each module, the inverse kinematics can be established with respect to the lengths of the chambers. It is to be noted that the given model does not consider the effects of gravity. In reality the base module and the tip module will deform differently, since the base module carries the extra weight of the tip module.

\section{Vi. Performance of the Robot}

The soft robot promises to provide high dexterity especially in constrained environments thanks to its design and kinematics.Fig. 6 gives an idea of the robot's workspace. The base module (blue) is fixed in one orientation in the figure. The backbone is constructed using the constant curvature model described in Section 5. The tip module (red), starts from the end of the base module and can be bent in all directions based on the chamber activation. The figure shows just some possible module configurations. If we consider all pressure combinations, the end of the tip module will trace a domelike shape. The modules can also elongate, so these domes
TABLE I

REPEATABILITy ANALYSIS AND MODEL COMPARISON FOR BENDING

\begin{tabular}{c|c|c|c|c} 
& $\begin{array}{c}\text { Simulated } \\
\text { bending } \\
\theta_{\text {sim }}(\mathrm{Deg})\end{array}$ & $\begin{array}{c}\text { Mean } \\
\text { bending } \\
\theta_{\text {act }} \\
\text { (Deg) }\end{array}$ & $\begin{array}{c}\text { Standard } \\
\text { deviation } \\
\text { (Deg) }\end{array}$ & $\begin{array}{c}\text { Error } \\
(\%)\end{array}$ \\
\hline $\begin{array}{c}\text { Single } \\
\text { Chamber } \\
\text { Activation }\end{array}$ & 148 & 154 & 0.25 & 3.8 \\
\hline $\begin{array}{c}\text { Two } \\
\text { Chamber } \\
\text { Activation }\end{array}$ & 167 & 171 & 0.34 & 2.3 \\
\hline
\end{tabular}

present a thickness wherein it may be oriented with multiple redundancies. Hence, the robot can avoid obstacles and reach the same targets in multiple configurations.

Since the manipulator is based on soft materials, the repeatability of the modules' deformation when subjected to the same input of pressure was studied. This repeatability was tested for one module at a time. Fig. 7-a shows a module while one of the three pair of chambers is inflated. In line with the assumption of the elongation being proportional to applied pressure, a constant curvature bending deformation is generated, as visible in the figure. Tests were conducted measuring the bending angle, i.e. the angle subtended by the tip of the module to a line passing from the original axis of the module (Fig 7-c and d). The maximum pressure applied to a pair of chambers was 1.5 bars. A ground truth measuring system (NDI Aurora [http://www.ndigital.com/medical/products/aurora/]), provided with sensing probes (Aurora Mini 6DOF Sensor $1.8 \mathrm{~mm}$ x 9 $\mathrm{mm}$ ) was used to record the position of the module's tip with to respect to the base. The trials were conducted in two sets, activation of a single pair of chambers (Fig. 7-c) and two pairs (Fig. 7-d). A total of ten trials were conducted for each pair of chambers actuation set to assess the repeatability. As expected the bending angle $\theta$ is higher when two pair of chambers are activated. As can be evinced from Fig. 7-c and d, the modules show a high degree of repeatability as represented by standard deviation values in the range of 0.3 . In order to test the validity of the kinematic model in describing the system, the bending angle of the tip was also calculated via the kinematic model, mentioned as $\theta_{\text {sim }}$.

The above discussed features, namely compliant nature for safe interaction and high dexterity can be put to practical use in the surgical endoscopic environment as an alternative to the rigid tools. As a case study, this paper reports the application of the robot as a camera module. The use of rigid tools limits the access of the camera to the line of insertion of the endoscope (Fig. 8). If the areas of interest are further away, as shown by a rod target, the rigid endoscope may not be able to reach them unless other entry points are created (Fig. 8-b and c). The soft robot can be moved with infinite possibilities in such a case. It may be able to 'snake' around obstacles to the site of interest (Fig. 8-d and e).

In order to test advantages offered by a soft robot in the actual surgical environment, the soft robot was tested by a team of surgeons in a human cadaver test. The procedure conducted was a total mesorectal excision (TME) in a cadaver model. The 




Fig. 7. Repeatability measurement setup and results. a) Single module bending with Constant Curvature. b) Bent configuration of the tip module using constant curvature model. c) Average trace of the tip with single chamber activation in black. Shape of the module is represented by the thick red curve and d)Average trace of tip with double chamber activation in black and shape of the module is represented by the thick red curve

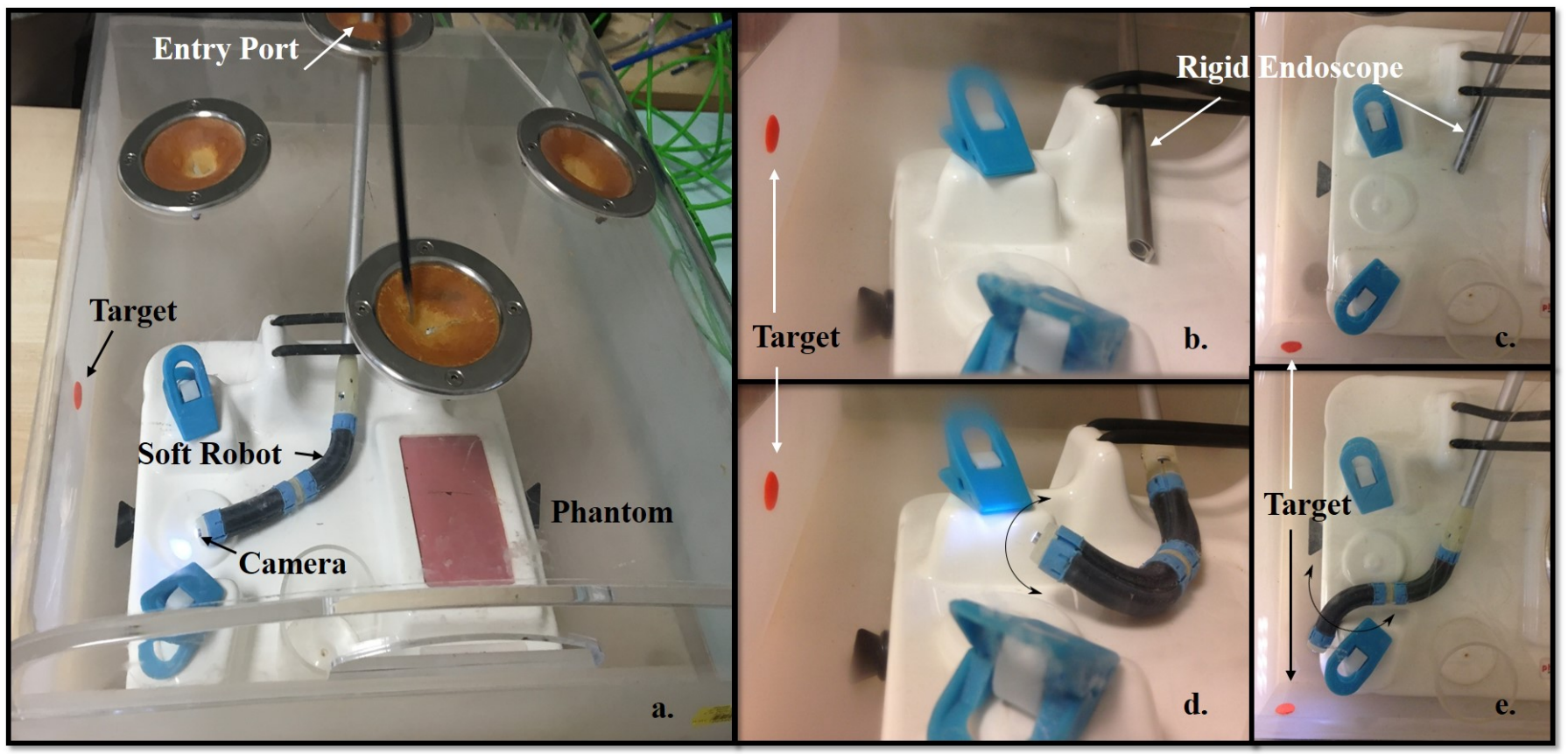

Fig. 8. Robot capabilities in constrained spaces. a) Overview of laparoscopic phantom. b) and c) show limitations of rigid tools to reach the target site. d) and e) represent the ability of the soft robot to reach the target site and still afford motion capabilities.

soft robot provided visual guidance, as described in more detail in [35]. Once inside the abdominal cavity, the soft robot was controlled by one surgeon using a joystick in a feed forward open loop manner.

Two monitors were used to follow the procedure: one was connected to the camera output of the soft robot (Misumi MD-T1003-65), while the other reported the view from a 30 degree optics from a standard laparoscope. This was used for documenting the motion of the soft robot (Fig. 9).

\section{DiscusSiON AND CONCLUSION}

MIS environments pose some unique challenges in terms of instrument design. Traditional tools are rigid, and have limited DOFs, making navigation and manipulation very difficult. The proposed soft robot can be a great asset in overcoming these challenges. Design of the robot has been optimized to suit, and hence be compatible with, the standard surgical tools. Being completely soft it poses very little danger of causing contact trauma. In this sense, it may help relieve stress levels of both the patient, and the surgeon since the surgeon may not have to fear damage to the environment. Additionally, it provides omni-directional motion that is not possible through standard tools. Design optimization not only satisfies the surgical environment constraints, but also increases the robot's effectiveness. A double cylinder chamber configuration provides a larger moment arm when activated due to the effective center of pressure of the cylinders being further away from the main axis in comparison to the use of one large cylinder, resulting in a stable bending motion. Fabrication of soft structures is often challenging since defects may result in actuators that are not repeatable. However, the resulting robot has shown to have good repeatability within a pressure working range of $0-1.5$ bars.

A distributed effect of the pressure about the robot's body 
a.

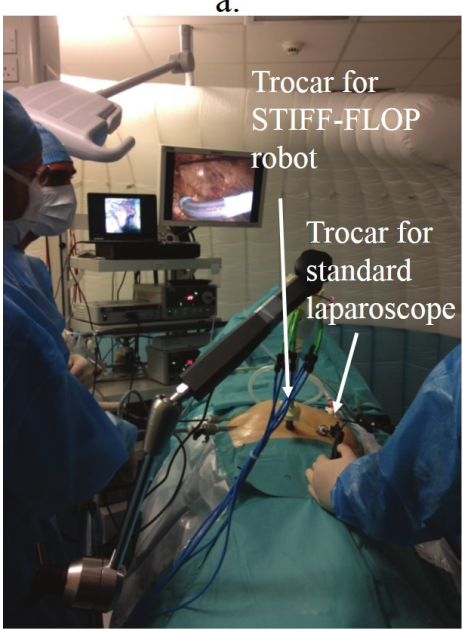

b.

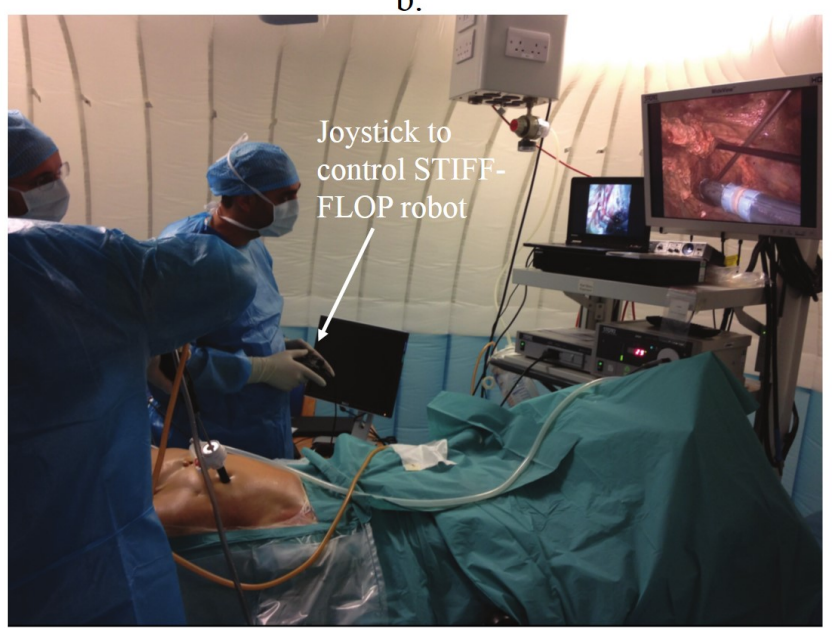

C.

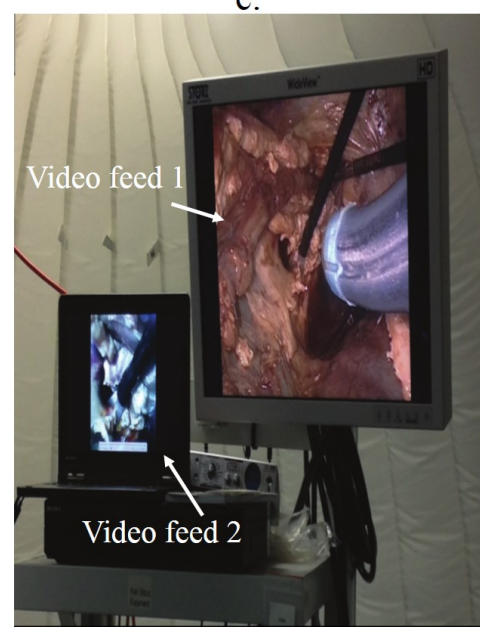

Fig. 9. Cadaver tests. a) The operating environment, showing the two trocars used for the STIFF-FLOP robot and a standard surgical endoscope. b) Another view showing the surgeon operating STIFF-FLOP by a joystick. c) Images from the two screens used during the operation. The video feed 1 is from the standard instrument and acts as visual feedback for control of the soft robot. Video feed 2 is provided by the soft robot which gave the surgeon better views of the operational site which was situated at a very challenging location for straight and stiff instruments.

results in a constant curvature motion, allowing the use of $\mathrm{CC}$ models for the kinematics. The $\mathrm{CC}$ approach provides a good estimate of the behavior of the robot, and could be used for formulating a controller. The influence of forces still need to be implemented in order to compensate for gravity and external interaction forces. However, the model is sufficient for the current level of control by the surgeon due to the visual feedback allowing steering via "open loop" position control.

The robot can navigate around organs to reach targets not directly in the line of sight of the trocar. In these regards, it can be a great tool for laparoscopic procedures. Current laparoscopic devices have actuation limited only to the end effectors. More than often, this actuation is also limited to one or two degrees of freedom. It is difficult to follow complex anatomical regions with such tools. The soft robot's efficacy was tested via a cadaver test where the robot was able to slide along anatomical features and provide a close up visualization of the surgical site at a very critical step of a TME procedure. It should be noted here that the strengths of the soft robot are in reaching hard to access regions. The camera system is only one of the possible uses. The lumen may house other tools apart from the current camera, and hence the robot may serve additional functions such as acting as an access channel for hard to reach anatomic regions. In this case the operation characteristics will differ, subject to the tool characteristics and the material in the lumen.

\section{ACKNOWLEDGMENT}

The authors would like to thank the STIFF-FLOP consortium for the useful suggestions and discussions related to the design of the surgical module.

\section{CONFLICT OF INTEREST}

The authors declare that there is no conflict of interest associated with this manuscript.

\section{REFERENCES}

[1] T. Robinson and G. Stiegmann, "Minimally invasive surgery." Endoscopy, 2004, vol. 36, no. 1, pp. 48-51.

[2] L. Vyas, D. Aquino, C.-H. Kuo, J. S. Dai, and P. Dasgupta, "Flexible robotics," BJU international, 2011, vol. 107, no. 2, pp. 187-189.

[3] C.-H. Kuo, J. S. Dai, and P. Dasgupta, "Kinematic design considerations for minimally invasive surgical robots: an overview," The International Journal of Medical Robotics and Computer Assisted Surgery, 2012, vol. 8, no. 2, pp. 127-145.

[4] J. Leroy, F. Jamali, L. Forbes, M. Smith, F. Rubino, D. Mutter, and J. Marescaux, "Laparoscopic total mesorectal excision (tme) for rectal cancer surgery: long-term outcomes," Surgical Endoscopy and Other Interventional Techniques, 2004, vol. 18, no. 2, pp. 281-289.

[5] M. Morino, U. Parini, G. Giraudo, M. Salval, R. B. Contul, and C. Garrone, "Laparoscopic total mesorectal excision: a consecutive series of 100 patients," Annals of surgery, 2003, vol. 237, no. 3, p. 335.

[6] http://www.intuitivesurgical.com/products/871145_Instrument Accessory_\%20Catalog.pdf. [Online]. Available: http: //www.intuitivesurgical.com/products/871145_Instrument_Accessory_\% 20Catalog.pdf

[7] J. Shang, D. P. Noonan, C. Payne, J. Clark, M. H. Sodergren, A. Darzi, and G.-Z. Yang, "An articulated universal joint based flexible access robot for minimally invasive surgery," in IEEE International Conference on Robotics and Automation (ICRA), 2011, pp. 1147-1152.

[8] J. Shang, C. J. Payne, J. Clark, D. P. Noonan, K. W. Kwok, A. Darzi, and G. Z. Yang, "Design of a multitasking robotic platform with flexible arms and articulated head for minimally invasive surgery," in IEEE International Conference on Intelligent Robots and Systems (IROS), 7-12 Oct. 2012, pp. 1988-1993.

[9] http://www.transenterix.com/technology/surgibot/. [Online]. Available: http://www.transenterix.com/technology/surgibot/.

[10] A. Tzemanaki, P. Walters, A. G. Pipe, C. Melhuish, and S. Dogramadzi, "An anthropomorphic design for a minimally invasive surgical system based on a survey of surgical technologies, techniques and training," The International Journal of Medical Robotics and Computer Assisted Surgery, 2014, vol. 10, no. 3, pp. 368-378, rCS-13-0041.R2.

[11] N. Simaan, K. Xu, W. Wei, A. Kapoor, P. Kazanzides, R. Taylor, and P. Flint, "Design and integration of a telerobotic system for minimally invasive surgery of the throat," The International Journal of Robotics Research, 2009, vol. 28, no. 9, pp. 1134-1153.

[12] A. H. Gosline, N. V. Vasilyev, A. Veeramani, M. Wu, G. Schmitz, R. Chen, V. Arabagi, P. J. del Nido, and P. E. Dupont, "Metal mems tools for beating-heart tissue removal," in IEEE International Conference on Robotics and Automation (ICRA), 2012, pp. 10.1109/ICRA.2012.6225210- 
[13] J. Burgner, P. J. Swaney, R. A. Lathrop, K. D. Weaver, and R. J. Webster, "Debulking from within: A robotic steerable cannula for intracerebral hemorrhage evacuation," IEEE Transactions on Biomedical Engineering, 2013, vol. 60, no. 9, pp. 2567-2575.

[14] J. Burgner, D. C. Rucker, H. B. Gilbert, P. J. Swaney, P. T. Russell, K. D. Weaver, and R. J. Webster, "A telerobotic system for transnasal surgery," IEEE/ASME Transactions on Mechatronics, 2014, vol. 19, no. 3, pp. 996-1006.

[15] M. Cianchetti, T. Ranzani, G. Gerboni, T. Nanayakkara, K. Althoefer, P. Dasgupta, and A. Menciassi, "Soft robotics technologies to address shortcomings in today's minimally invasive surgery: the stiff-flop approach," Soft Robotics, 2014, vol. 1, no. 2, pp. 122-131.

[16] A. Shiva, A. Stilli, Y. Noh, A. Faragasso, I. D. Falco, G. Gerboni, M. Cianchetti, A. Menciassi, K. Althoefer, and H. A. Wurdemann, "Tendon-based stiffening for a pneumatically actuated soft manipulator 2016," IEEE Robotics and Automation Letters, vol. 1, no. 2, pp. 632637.

[17] H. Wurdemann, A. Stilli, and K. Althoefer, "An antagonistic actuation technique for simultaneous stiffness and position contro," in International Conference on Intelligent Robotics and Applications (ICIRA), vol. 1, 2015, pp. 164-174.

[18] F. Maghooa, A. Stilli, Y. Noh, K. Althoefer, and H. A. Wurdemann, "Tendon and pressure actuation for a bio-inspired manipulator based on an antagonistic principle," in 2015 IEEE International Conference on Robotics and Automation (ICRA), May 2015, pp. 2556-2561.

[19] A. Stilli, H. A. Wurdemann, and K. Althoefer, "Shrinkable, stiffnesscontrollable soft manipulator based on a bio-inspired antagonistic actuation principle," in IEEE International Conference on Intelligent Robots and Systems (IROS), Sept 2014, pp. 2476-2481.

[20] H. Abidi and M. Cianchetti, "On intrinsic safety of soft robots," Frontiers in Robotics and AI, 2017, vol. 4, p. 5.

[21] K. Suzumori, S. Iikura, and H. Tanaka, "Flexible microactuator for miniature robots," IEEE Micro Electro Mechanical Systems (MEMS), 1991, pp. 204-209.

[22] K.Suzumori, S. Iikura, and H. Tanaka, "Applying a flexible microactuator to robotic mechanisms," IEEE Control Systems, 1992, vol. 12, no. 1, pp. 21-27.

[23] E. T. Roche, M. A. Horvath, I. Wamala, A. Alazmani, S.-E. Song, W. Whyte, Z. Machaidze, C. J. Payne, J. C. Weaver, G. Fishbein, J. Kuebler, N. V. Vasilyev, D. J. Mooney, F. A. Pigula, and C. J. Walsh, "Soft robotic sleeve supports heart function," Science Translational Medicine, 2017, vol. 9, no. 373.

[24] D. Rus and M. T. Tolley, "Design, fabrication and control of soft robots," Nature, 2015, vol. 521, no. 7553, pp. 467-475.

[25] T. Ranzani, G. Gerboni, M. Cianchetti, and A. Menciassi, "A bioinspired soft manipulator for minimally invasive surgery," Bioinspiration \& biomimetics, 2015, vol. 10, no. 3, p. 035008.

[26] G. Gerboni, M. Brancadoro, G. Tortora, A. Diodato, M. Cianchetti, and A. Menciassi, "A novel linear elastic actuator for minimally invasive surgery: development of a surgical gripper," Smart Materials and Structures, 2016, vol. 25, no. 10, p. 105025.

[27] J. Fras, J. Czarnowski, M. Macias, J. Glowka, M. Cianchetti, and A. Menciassi, "New stiff-flop module construction idea for improved actuation and sensing," in IEEE International Conference on Robotics and Automation (ICRA). IEEE, 2015, pp. 2901-2906.

[28] M. Cianchetti, T. Ranzani, G. Gerboni, I. De Falco, C. Laschi, and A. Menciassi, "Stiff-flop surgical manipulator: mechanical design and experimental characterization of the single module," in IEEE International Conference on Intelligent Robots and Systems (IROS). IEEE, 2013, pp. 3576-3581.

[29] S. Sareh, Y. Noh, M. Li, T. Ranzani, H. Liu, and K. Althoefer, "Macrobend optical sensing for pose measurement in soft robot arms," Smart Materials and Structures, 2015, vol. 24, no. 12, p. 125024.

[30] A. W. Mahoney, T. L. Bruns, P. J. Swaney, and R. J. Webster, "On the inseparable nature of sensor selection, sensor placement, and state estimation for continuum robots or 'where to put your sensors and how to use them'," in IEEE International Conference on Robotics and Automation (ICRA), May 2016, pp. 4472-4478.

[31] G. Gerboni, A. Diodato, G. Ciuti, M. Cianchetti, and A. Menciassi, "Feedback control of soft robot actuators via commercial flex bend sensors," IEEE Transactions on Mechatronics, 2017, vol. PP, no. 99, pp. $1-1$.

[32] T. C. Searle, K. Althoefer, L. Seneviratne, and H. Liu, "An optical curvature sensor for flexible manipulators," in IEEE International Conference on Robotics and Automation (ICRA), May 2013, pp. 4415-4420.
[33] R. J. Webster and B. A. Jones, "Design and kinematic modeling of constant curvature continuum robots: A review," The International Journal of Robotics Research, 2010, vol. 29, pp. 1661-1683.

[34] S. Neppalli, M. A. Csencsits, B. A. Jones, and I. Walker, "A geometrical approach to inverse kinematics for continuum manipulators," in IEEE International Conference on Intelligent Robots and Systems (IROS), 2008, pp. 3565-3570.

[35] A. Arezzo, Y. Mintz, M. E. Allaix, S. Arolfo, M. Bonino, G. Gerboni, M. Brancadoro, M. Cianchetti, A. Menciassi, H. Wurdemann, Y. Noh, K. Althoefer, J. Fras, J. Glowka, Z. Nawrat, G. Cassidy, R. Walker, and M. Morino, "Total mesorectal excision using a soft and flexible robotic arm: a feasibility study in cadaver models," Surgical Endoscopy, 2017, vol. 31 , no. 1 , pp. $264-273$. 